\title{
Fredkin Incentive Match
}

Pittsburgh, PA

August 15-17, 1982

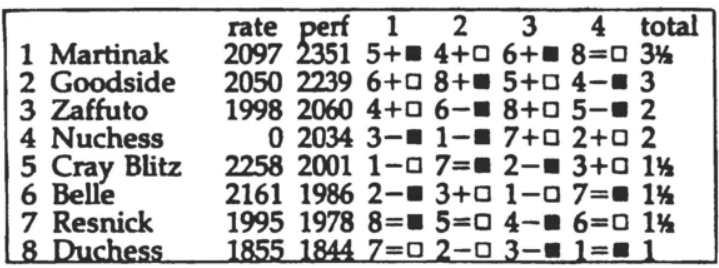

Cray Blitz - Martinak

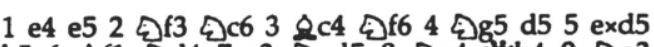

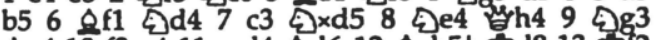

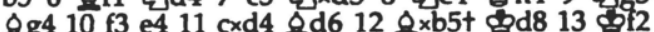

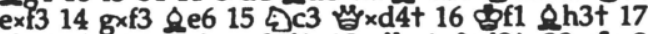

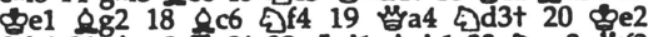

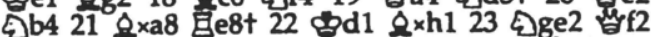

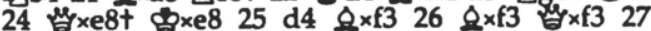

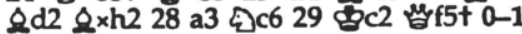

$$
\text { Duchess - Resnick }
$$

1 e4 e5 2 f3 0 f6 3 d4 $5 \times 4 \times 4$ od3 d5 5 किxe5 Qe7 6 O-O O-O 7 c4 \&66 8 c5 c6 9 I le1 2 bd7

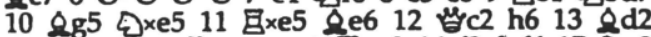

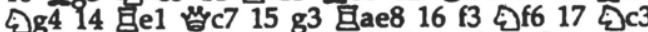
Q

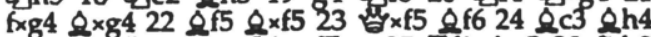

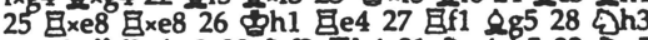

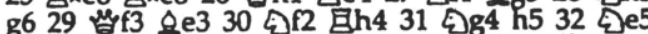

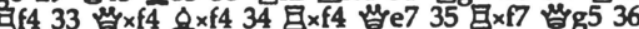

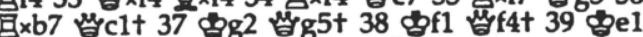

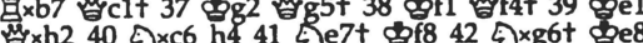
43 e5 $\mathrm{mg} 1+44 \mathrm{gd} 2 \mathrm{~h} 345 \mathrm{G} \times \mathrm{a} 7 \mathrm{~h} 246 \mathrm{Ga}+$

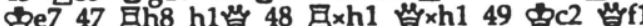
50 b4 Me2t 51 bb3 Md1t 52 bb2

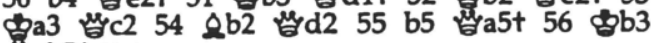
$\mu \mathrm{g} \times \mathrm{b} 5 \mathrm{t} / \mathrm{y}-\mathrm{k} / \mathrm{2}$

$$
\text { Zaffuto - Nuchess }
$$

1 d4 छf6 2 घf3 d5 3 c4 dxc4 4 a4 \&e6 5 घc3 Qc6 6 \&g5 \&ff5 7 a5 h6 8 \&.f6 exf6 9 d5 5 b4 10 4 ㅆe7 11 oxc4 oxe4 12 O-O f5 13 a6 bxa6 14

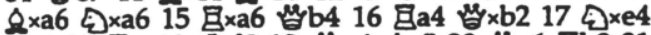

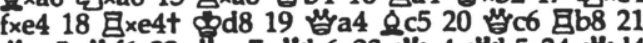
M. $\times c 5$ M. f6 25 Gfe1 Mud7 26 h3 ab7 27 kal bc8 28

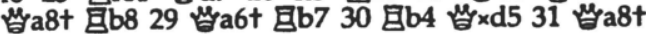
1-0

$$
\text { Goodside - Belle }
$$

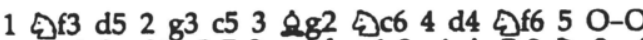

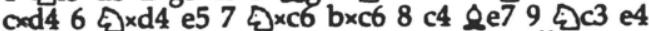
10 o 5 O-O 11 cd5 $2 \times d 512$ oxe7 $\mu_{\times \times e 7} 13$

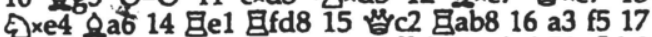

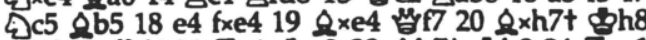

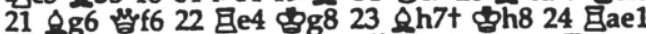

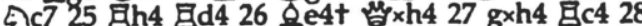

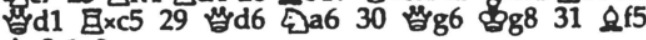
Q $\mathrm{e} 2$ 1-0
Martinak - Nuchess

1 d4 ¿f6 2 ¿f3 d5 3 c4 dxc4 4 a4

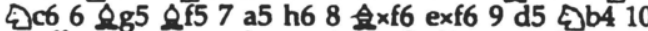
e4 He7 11 oxc4 $0 \times e 412$ O-O f5 13 a6 bxa6 14

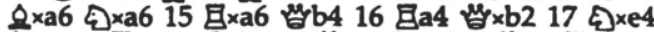

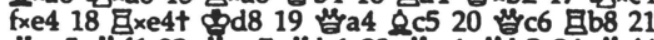

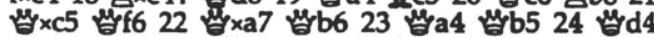

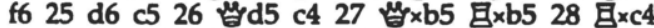

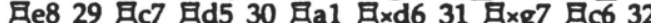

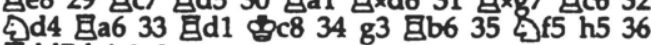
Gdd7 h4 1-0

$$
\text { Resnick - Cray Blitz }
$$

1 e4 c5 2 \&c3 e6 3 g3 d5 4 exd5 exd5 5 d4 cxd4 6

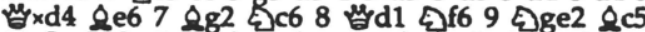
10 O-O O-O 11 o,5 0 e7 12 oxf6 oxf6 13 Oxd5 Qxd5 14 ․ㅏ․

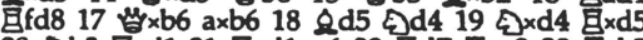

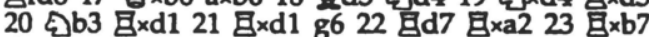

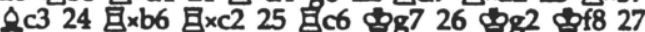
5d4 目d2 28 \&b3 目c2 29 目c7 be8 30 हd4 目d2

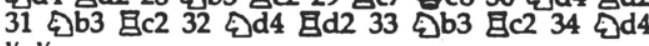
मे-

$$
\text { Duchess - Goodside }
$$

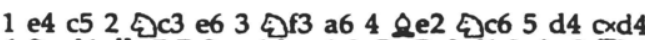

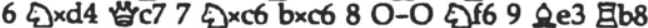
10 b3 0 b4 11 쌈3 O-O 12 Oa4 d5 13 Oc5 Hd8

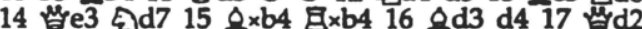

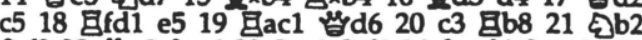

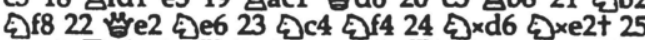
Qxe2 Ixd6 26 Id2 0 b7 27 \&3 Ibd8 28 oc4 bf8

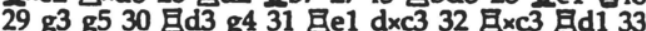
bf2 E1d2+ 34 Ge2 h5 35 a3 I8d4 36 be3 ad1

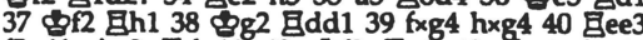

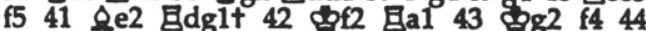

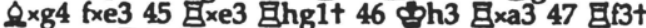

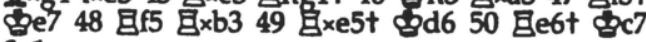
0-1

$$
\text { Belle - Zaffuto }
$$

1 e4 c5 2 c3 d5 3 exd5 kyxd5 4 d4 e5 5 dxe5

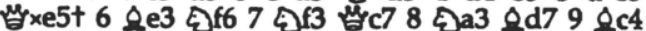

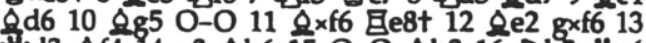

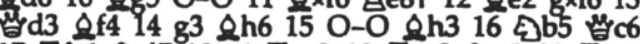
17 Ife1 18 d7 18 axe2 19 Exe2 0 e5 20 Exes

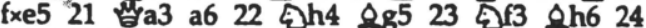

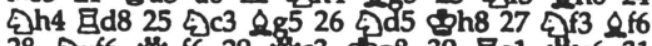

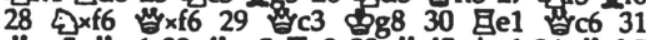

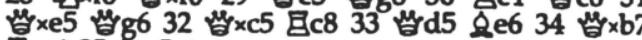
Oxc4 35 \& $251-0$ 


\section{Goodside - Cray Blitz}

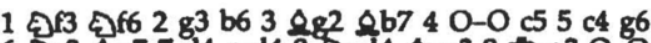

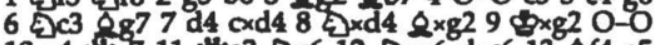

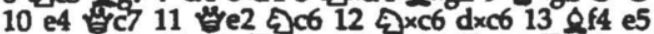

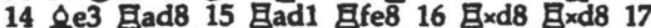

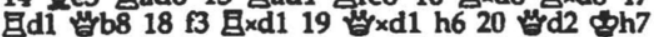
21 g4 b5 22 cxb5 ob5 23 읃 b4 24 छdd5 a5 25

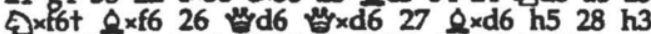

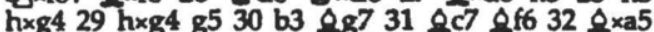

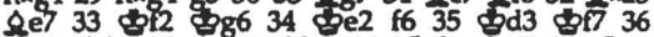

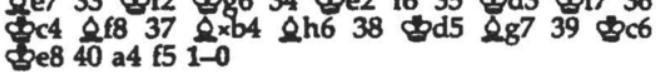

\section{Nuchess - Resnick}

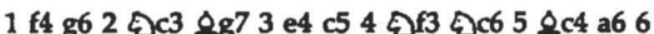
O-O e6 7 a4 5 ge7 8 e5 d5 9 exd6 $\mu$. $x$ d6 10 Oe4

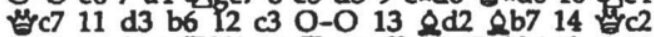

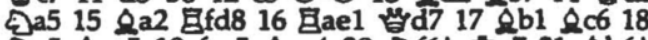

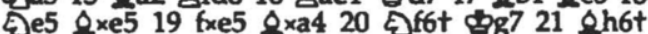

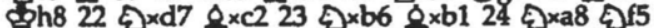

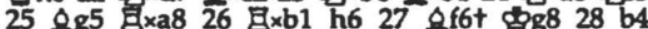

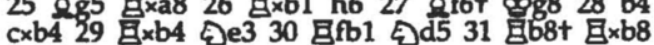
$32 \mathrm{O} \times \mathrm{b} 8+1-0$

$$
\text { Zaffuto - Duchess }
$$

1 d4 d5 2 c4 e6 3 \&c3 \& 864 \&g5 \&e7 5 e3 O-O 6 cod5 exd5 7 od3 c6 8 rrc2 5 bd7 9 दोge2 Ie8 10 O-O \&f8 11 Eabl og 12 b4 0 dd 13 h3

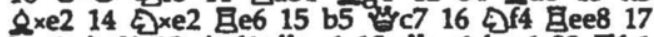

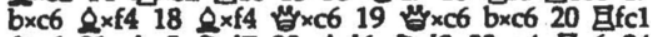

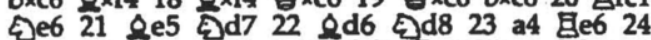

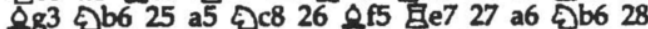

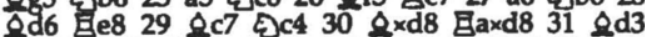

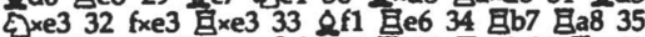

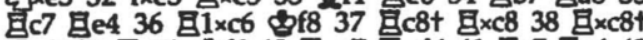

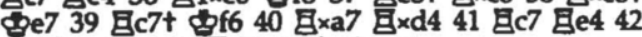

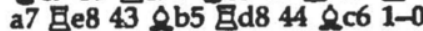

$$
\text { Belle - Martinak }
$$

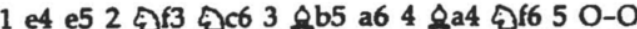

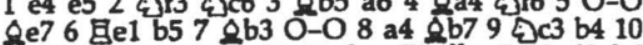

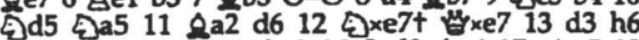
14 c3 bxc3 15 bxc3 o c8 16 \&d2 0 e6 17 c4 c5 18

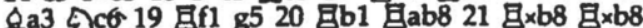

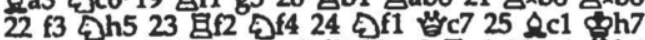
26 仓 x44 gxf4 27 Qd2 0 d7 28 g3 Ig8 29 g4

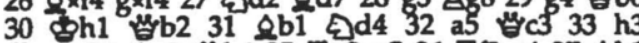

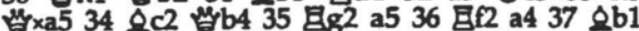

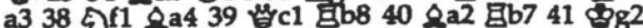

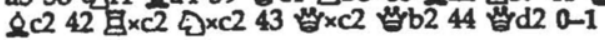

$$
\text { Martinak - Duchess }
$$

1 d4 d5 2 c4 dxc4 3 घु3 छीf6 4 घीc3 a6 5 a4 छिc6

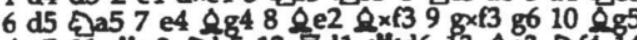

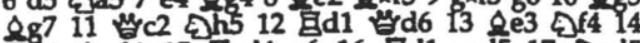
Od4 $0 \times d 4 \quad 15$ Ixd4 c6 16 Id1 cxd5 17 \&xd5

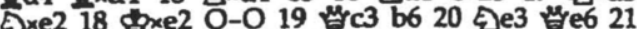
h4 Efe8 22 घd5 Hdd6 23 h5 Ilec8 24 f4 4 b3 25

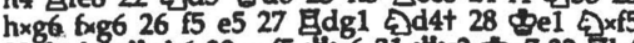

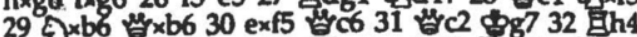

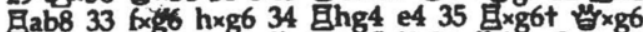

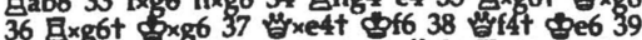

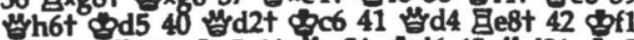

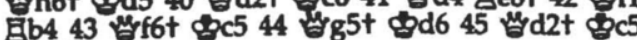

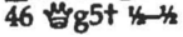

$$
\text { Cray Blitz - Zaffuto }
$$

1 e4 c5 2 \&13 e6 3 d4 oxd4 4 Exd4 a6 5 घc3

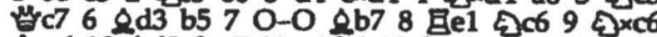
oxc6 10 요d2 \& 14 \& $\times c_{3}$ g6 15 Qb5 $0 \times b 516$ axb5 $2 \mathrm{g7} 17 \mathrm{mb3}$

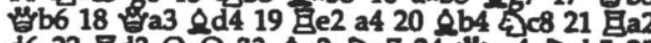

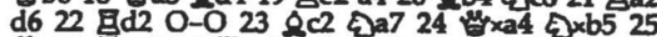
잘.

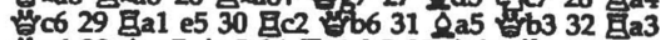

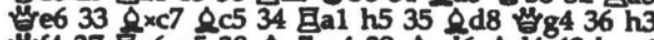

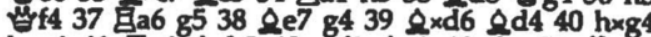

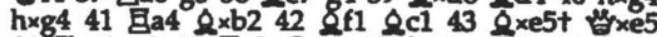

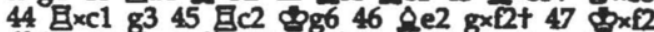

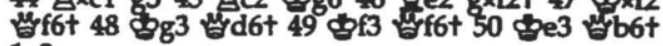
1-0

$$
\text { Nuchess - Goodside }
$$

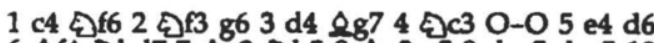
6 Qf4 छิbd7 7 \&e2 Qh5 8 \&e3 e5 9 dxe5 dxe5 10

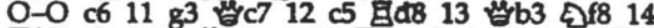

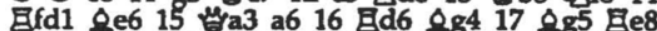

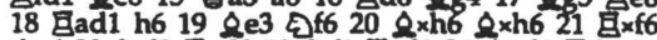

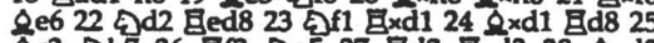

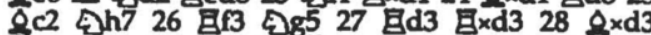

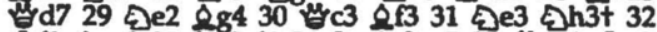

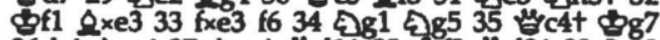

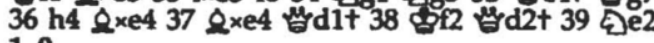
1-0

$$
\text { Resnick - Belle }
$$

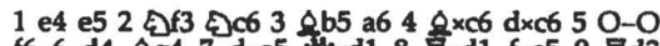

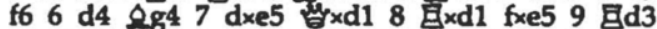

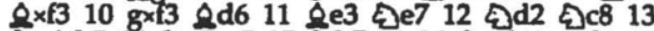

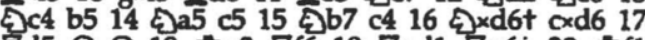

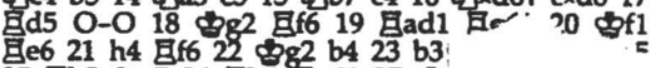

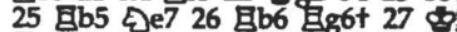
Ile6 29 Ed5 5 e7 30 Edb5 2863

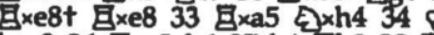

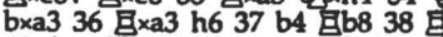

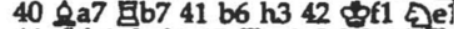

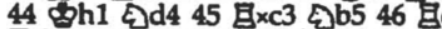
Id7 48 b7 $2 \times a 749$ If8+ bxf8 Hor 